\title{
Interaktion pulmonaler Epithelzellen mit Streptococcus pneumoniae
}

\author{
Pneumococci - Epithelial Interaction
}

Die ambulant erworbene Pneumonie ist die häufigste Infektionskrankheit in den Industrienationen und ist die dritthäufigste Todesursache weltweit. Mit Abstand die meisten Pneumonien rufen Infektionen mit Pneumokokken hervor. Pneumonien ausgelöst durch $S$. pneumonie sind u.a. durch eine massive Entzündungsreaktion geprägt, welche ständig das Risiko einer überschießenden pulmonalen Inflammation mit folgendem Organversagen in sich trägt. Obwohl pulmonale Epithelzellen primäre Zielzellen invadierender Pathogene (einschließlich Pneumokokken) darstellen, liegen kaum Informationen über die Pneumokokken-Epithelzellinteraktion in der Lunge vor.

Wir untersuchen daher molekulare Mechanismen der Interaktion von Pneumokokken mit pulmonalen Epithelzellen und analysieren involvierte Signalwege. Mittelfristig kann das Verständnis dieser Signalprozesse rationale Grundlagen zur Entwicklung innovativer Therapiestrategien der Pneumonie legen.

Wesentlich für die Stimulation der angeborenen Immunität ist das Erkennen spezieller hochkonservierter Strukturen der Erreger durch Keimbahn-kodierte Wirtsrezeptoren („Pathogen recognition receptors“, PRR). Pulmonale Epithelzellen detektieren Pneumokokken oder ihre Bestandteile durch die membranständigen PRR-Rezeptoren TLR1, 2 und 4, wobei TLR1 und 2 synergistisch wirkten [1]. Pneumokokkeninfektionen gehen mit der Invasion pulmonaler Zellen durch das Pathogen einher. Wir identifizierten das „Nucleotide-binding oligomerization domain protein-2“ (Nod2) als einen zytosolischen PRR für intrazelluläre Pneumokokken in Epithelzellen [2]. Infolge beobachteten wir eine vermehrte Freisetzung verschiedener CC und CXC, sowie Th1 and Th2 Zytokine und von Wachstumsfaktoren durch infi-

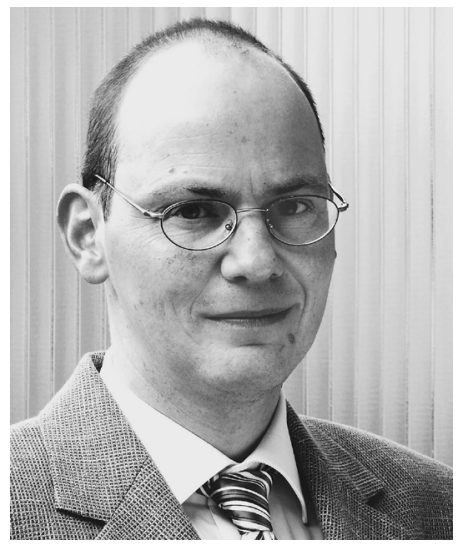

PD Dr. med. Stefan Hippenstiel

zierte Epithelzellen [2-4]. Somit können pulmonale Epithelzellen sowohl extra- als auch intrazelluläre Pneumokokken detektieren und durch die Freisetzung von Zytokinen aktiv in die Entzündungsreaktion eingreifen.

Um Einblick in die der Zellaktivierung zugrunde liegenden Mechanismen zu gewinnen, analysierten wir verschiedene Signaltransduktionswege in Pneumokokken infizierten pulmonalen Epithelzellen am Beispiel der Interleukin-8 (IL-8) Bildung. Besondere Aufmerksamkeit ruhte auf den Mitogen-aktivierten Kinasen (MAPK) und Transkriptionsfaktoren (NF- $\mathrm{B}, \mathrm{AP}-1$ ).

Wir fanden eine Aktivierung der p38 MAPK, ERK sowie JNK Kinase in Pneumokokken exponiertem Epithel in vitro und in infizierten Mäuselungen $[3,4]$. Blockade der p38 MAPK hemmte die Pneumokokken induzierte Bildung verschiedener Zytokine [4]. 
Detaillierte Analysen ergaben, dass Pneumokokken den kanonischen Signalpfad zur NF- $\mathrm{kB}$ Aktivierung in vitro und in vivo stimulieren [4]. Das kleine GTP bindende Rho Protein Rac1 wirkte dabei als ein wichtiges Schaltmolekül zur TLR abhängigen NF- $\mathrm{KB}$ Stimulation in Pneumokokken infiziertem Epithel [1]. Die Adaptormoleküle IRAK, IRAK2, TRAF6, NIK, TAB2 und TAK1 konnten erstmals als Signalintermediate Pneumokokken assoziierter, Nod2 abhängiger NF-kB Aktivierung in pulmonalem Epithel identifiziert werden [2].

Interessanterweise konvergierten der p38 MAPK Signalpfad mit NF- $\mathrm{kB}$ abhängiger Transkription von Zytokinen auf Promotorebene in pulmonalen Epithelzellen [4]: Am IL-8 Promotor fand nur eine Bindung der mRNA Polymerase II (und folgende Gentranskription) statt, wenn zuvor durch die p38 MAPK eine Phosphorylierung der NF- $\mathrm{KB}$ Untereinheit p65/RelA an Serin 536 erfolgt war. Eine ähnlich komplexe Regulation erbrachten Untersuchungen zur Regulation Pneumokokken induzierter Cyclooxygenase-2 Expression in pulmonalem Epithel [5].

Langfristig konnte die Exposition kultivierter pulmonaler Epithel- [6] und Endothelzellen [7] gegenüber Pneumokokken zur Apoptose führen. Neben insbesondere Caspase- 6 vermitteltem programmiertem Zelltod fand sich auch Caspase-unabhängige Apoptose. Sowohl die Erhöhung intrazellulärer cAMP Spiegel als auch vermehrte Expression des anti-apoptotischen Moleküls Bcl-2 blockierte Pneumokokken assozierte Apoptose in vitro.

Offensichtlich wirken pulmonale Epithelzellen also nicht nur als eine passive Barriere bei (Pneumokokken-) Pneumonie, sondern sie tragen aktiv zur Orchestrierung der Immunantwort bei. Die derzeitigen Studien bieten einen ersten orientierenden Einblick in die Komplexität der pulmonalepithelialen Interaktion mit Pathogenen und machen zugleich deutlich, welch große Wissenslücken im klinisch bedeutenden Krankheitsbild der Pneumonie bestehen. Neben vertiefenden Untersuchungen zur Pathogenese der Pneumokokkenpneumonie adressieren wir daher im Vergleich auch molekulare Mechanismen pulmonaler Legionelleninfektionen [8].

\section{Literatur}

${ }^{1}$ Schmeck B, Huber S, Moog K et al. Pneumococci induced TLR- and Rac1-dependent NF- $\mathrm{kB}$-recruitment to the IL-8 promoter in lung epithelial cells. In Revision,

${ }^{2}$ Opitz B, Püschel A, Schmeck B et al. Nucleotide-binding oligomerization domain proteins are innate immune receptors for internalized Streptococcus pneumoniae. J Biol Chem 2004; 279: 36426-36432

${ }^{3}$ Schmeck B, Moog K, Zahlten J et al. Streptococcus pneumoniae induced cJun-N-terminal kinase- and AP-1-dependent cytokine release by lung epithelial cells. Eingereicht.

${ }^{4}$ Schmeck B, Zahlten J, Moog K et al. Streptococcus pneumoniae induced p38 MAPK dependent phosphorylation of RelA at the interleukin-8 promotor. J Biol Chem 2004; 279: 53241 - 53247

${ }^{5}$ Dje N'Guessan P, Hippenstiel S, Ollomang M et al. Streptococcus pneumoniae induced p38 MAPK and NF- $\kappa$ B dependent COX-2 expression in human lung epithelium. Eingereicht

${ }^{6}$ Schmeck B, Gross R, N'Guessan PD et al. Streptococcus pneumoniaeinduced caspase 6-dependent apoptosis in lung epithelium. Infect Immun 2004; 72: 4940-4947

${ }^{7}$ N'Guessan DjeP, Schmeck B, Ayim A et al. Streptococcus pneumoniae R6x induced p38 MAPK and JNK-mediated caspase-dependent apoptosis in human endothelial cells. Thromb Haemost 2005; 94: $295-303$

${ }^{8}$ Schmeck B, Dje N'Guessan P, Ollomang M et al. Legionella pneumophila induced NF- $\mathrm{\kappa B}$ - and mitogen activated protein kinase-dependent cytokine release by alveolar epithelial cells. Eingereicht. 